\title{
Zeolite Modified Gas Sensors For Environmental Monitoring
}

\author{
A. Afonja ${ }^{1}$, I.P. Parkin ${ }^{1}, \underline{\text { R. Binions }}^{1,2}$ \\ ${ }^{1}$ Department of Chemistry, University College London, London WC1H OAJ, United Kingdom \\ ${ }^{2}$ School of Engineering and Materials Science, Queen Mary University of London, London E1 4NS, \\ United Kingdom \\ email: r.binions@qmul.ac.uk
}

\begin{abstract}
Metal oxide semiconductor gas sensors with zeolite over layers or admixtures have been prepared and used to investigate the detection of a number of environmentally important gases. The results show the sensors have consistent and repeatable gas response behavior. Additional testing indicated that the sensors gave good discrimination between gases across an array of sensors.
\end{abstract}

Key words: Metal Oxide Semiconductor Sensor, Zeolite, Environmental Monitoring.

\begin{abstract}
Introduction
This work is aimed at addressing the problem of complex vapor measurement in a repeatable and reliable manner. The main issue is producing an array of sensors that gives a significantly discriminatory response pattern. A large body of research has been produced in this area examining sensor material composition [1]. Large numbers of semiconducting metal oxides are gas sensitive at elevated temperatures [2] and relatively minor modifications to the material composition can have significant effects on the gas sensing properties of such materials, particularly where they cause a change in microstructure and or crystallite size.
\end{abstract}

Sensor gas response has also been shown to be drastically altered by the inclusion of a finely divided catalyst material over the surface of the metal oxide. The catalyst causes reactions at the surface of the material to take place and changes the local atmosphere and hence the local conductivity in a porous structure [3].

Another widely explored route is to operate similar devices at different temperatures [4,5]. In general however, there is usually a strong temperature dependence on the sensor response. As such the array can easily be dominated by the behavior of one or two sensors and any selectivity is lost. We have explored means of using the same semiconducting oxide sensor materials, operated at the same temperature and without any compositional modification. As such the effects of drift, poisoning and water vapour should be similar for all elements.

The basic concept of our approach is to consider the sensor device as having two parts: a sensing element, in this case porous tungsten trioxide or chromium titanium oxide (CTO) and a transformation element, in this case the hydrogen variants of various zeolites in order to modify or restrict the composition of the gas atmosphere that is able to interact with the sensor element. Zeolites are excellent for this as they are molecular size and shape specific as well as being able to perform various catalytic reactions.

We've previously shown the use of over layered zeolites as highly effective at both generating a discriminating array and producing selective individual sensors [6-8]. In this paper we report on the use of over-layered and admixed zeolites

\section{Experimental}

The Chromium titanium oxide was prepared as previously described [6]. $\mathrm{Cr}_{2} \mathrm{O}_{3}(19.525 \mathrm{~g}, 0.13$ mol, prepared by thermal decomposition of ammonium dichromate at $300^{\circ} \mathrm{C}$ ) was mixed with propan-2-ol (500 $\mathrm{mL})$ for 10 minutes using a homogeniser $(1000 \mathrm{rpm})$. Titanium isopropoxide $(1.954 \mathrm{~g}, 6.6 \mathrm{mmol})$ and water (5 $\mathrm{mL}$ in $50 \mathrm{~mL}$ propan-2-ol) were added to the suspension over 15 minutes and the mixture rotary evaporated in an ultrasonic bath.

The zeolite H-LTA was prepared by ion exchange of the $\mathrm{Na}$ zeolite form (obtained from Zeolyst International) with a $1 \mathrm{M} \mathrm{NH} 4 \mathrm{OH}$ 
solution for 12 hours at $60{ }^{\circ} \mathrm{C}$ followed by calcination for 12 hours at $500{ }^{\circ} \mathrm{C}$ [6]. ZSM-5 was obtained in $\mathrm{NH}_{4}$ form (also from Zeolyst international) and similarly calcined after being dried in air at $100^{\circ} \mathrm{C}$. These temperatures were chosen to ensure the total exclusion of water from the zeolite structure and to ensure complete conversion of the ammonium form to the active acid form. Zeolite $Y$ was obtained from Zeolyst in $\mathrm{H}-\mathrm{Y}$ form and was not subject to further processing. EDAX analysis was used to determine the zeolite $\mathrm{Si} / \mathrm{Al}$ ratios and the extent of the ion-exchange.

In preparation for screen-printing, inks were made by grinding the oxide and zeolite powders with an organic vehicle (ESL400) in a pestle and mortar. The inks were then screen-printed onto strips of $3 \times 3 \mathrm{~mm}$ alumina substrates with an integral platinum heater and gold electrode pattern [3]. Between each layer of print the sensors were dried under an infra-red lamp for 10 minutes.

The sensors consisted of metal oxide layers that were $\sim 50 \mu \mathrm{m}$ thick in total overlaid with layers of zeolite, again $\sim 50 \mu \mathrm{m}$ thick or by $\sim 100$ $\mu \mathrm{m}$ layers of metal oxide mixed with $30 \mathrm{wt} . \%$ zeolite. The thickness was measured using a depth gauge at five points along the strip of substrates to give an average. The sensors were fired at $600{ }^{\circ} \mathrm{C}$ in a Carbolite HTC 1400 furnace for 2 hours to burn off the organic vehicle.

Contacts to the devices were formed by spot welding $50 \mu \mathrm{m}$ diameter platinum wire to pads of the track material in the corner of the sensor chip. The sensor heater was kept at constant temperature by incorporating it into a constant resistance Wheatstone bridge. Gas sensing experiments were performed on a locally constructed test rig [3]. Test gas streams of environmentally important gases [9] were prepared by dilution of synthetic air $(79 \%$ nitrogen, $21 \%$ oxygen) containing ammonia (25 ppm) or butene, propene or ethene (all 50 ppm). All the devices were operated at $400^{\circ} \mathrm{C}$.

X-Ray diffraction (XRD) was carried out on a Bruker D8 Discover diffractometer with a CuKa1 source $(\lambda=1.5406 \AA)$ and a GADDS detector. Energy dispersive X-ray Spectroscopy (EDAX) was used to obtain elemental compositions using an Oxford Instruments INCA energy system in conjunction with a Phillips XL30 environmental scanning electron microscope (SEM). XPS measurements were performed on a VG Escalab 220. Top-down and cross-sectional SEM pictures were obtained using a JEOL $6301 \mathrm{~F}$ with an accelerating voltage of $15 \mathrm{kV}$.
Gold sputter-coating of the SEM samples was carried out on an Edwards S105B sputtercoater. The materials were screen-printed using a DEK 1202. Welding of the sensors was done on a MacGregor DC601 parallel gap resistance welder.

\section{Results}

X-ray diffraction analysis of the tungsten oxide (monoclinic $\mathrm{WO}_{3}$ ) and CTO (eskolite structure) was carried out before and after firing the sensors. It was found that the materials crystallinity remained intact with no sign of degradation. Scanning electron microscopy was conducted to examine the sensors microstructure (Fig 1.). In all cases the sensors were made up of loosely packed particulates around $1 \mu \mathrm{m}$ in diameter with some grains fused together.

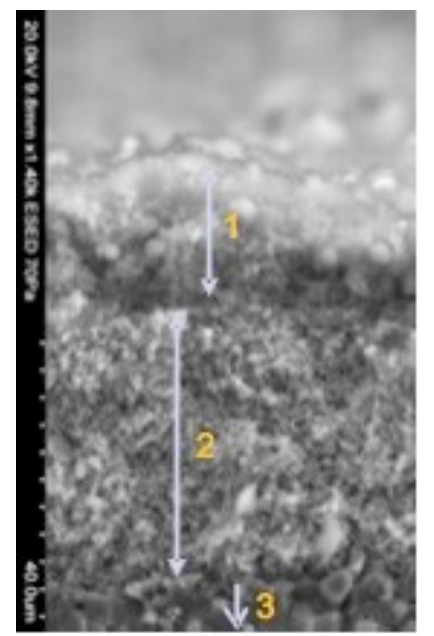

Fig. 1. Scanning electron micrograph showing typical crosssectional view of zeolite layered thick films. Regions 1, 2 and 3 demarcate zeolite $(\sim 62 \mu \mathrm{m}), \mathrm{WO}_{3}(\sim 168 \mu \mathrm{m})$ and alumina substrate boundaries respectively.

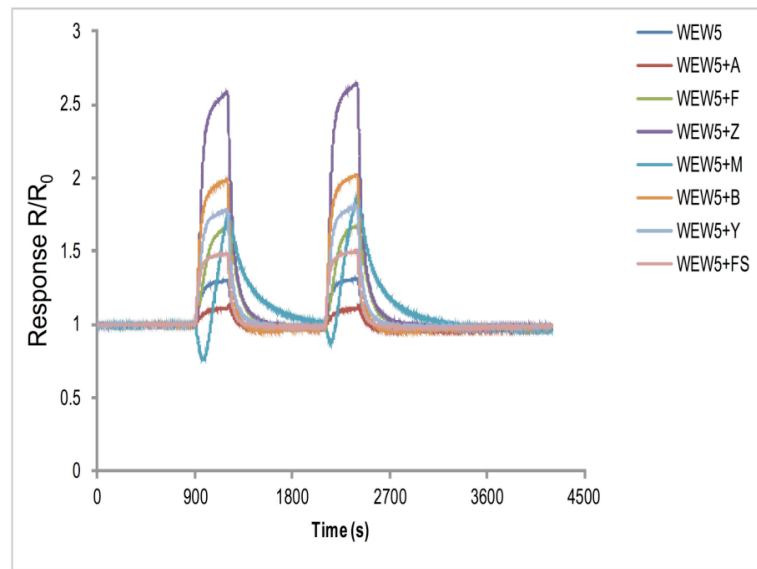

Fig. 2. Gas response behavior sensors with zeolite over-layers to $25 \mathrm{ppm} \mathrm{NH}_{3}$ in dry air at an operating temperature of $400{ }^{\circ} \mathrm{C}$. Where WEW5 is a tungsten trioxide sensor, over-layed with: $+A$ - zeolite $A,+F-$ ferrierite, $+Z-Z S M-5,+M-$ mordenite, $+B$ - zeolite $B$, $+Y$ - zeolite $Y,+F S$ - fumed silica. 
Overall the sensor microstructure was open and so gas is able to diffuse through and around the zeolite layers or particles before interacting with the sensor surface.

The control sensor response on exposure to 25 ppm of $\mathrm{NH}_{3}$ were as expected showing an increase in resistance (Fig. 2). The ammonium form of zeolites is generally an intermediate step in metal ion exchange reactions with the ammonium ion readily absorbing onto acidic zeolite sites, due to this affinity we can expect significant absorption on the most acidic zeolites tested - in this case zeolite A, which clearly has the lowest response.

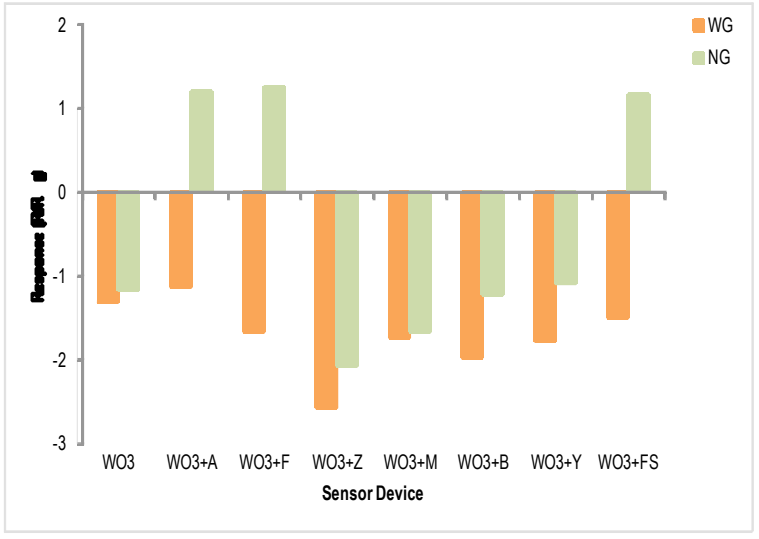

Fig. 3. Bar chart comparing response magnitudes of the control and overlaid WG and NG $\mathrm{WO}_{3}$ sensor

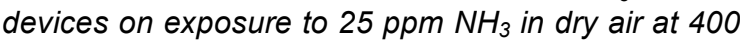
${ }^{\circ} \mathrm{C}$. The chart indicates the presence of oxidant products of $\mathrm{NH}_{3}$ combustion diffusing into the bulk of the $A$, ferrierite and fumed silica layered NG sensors.

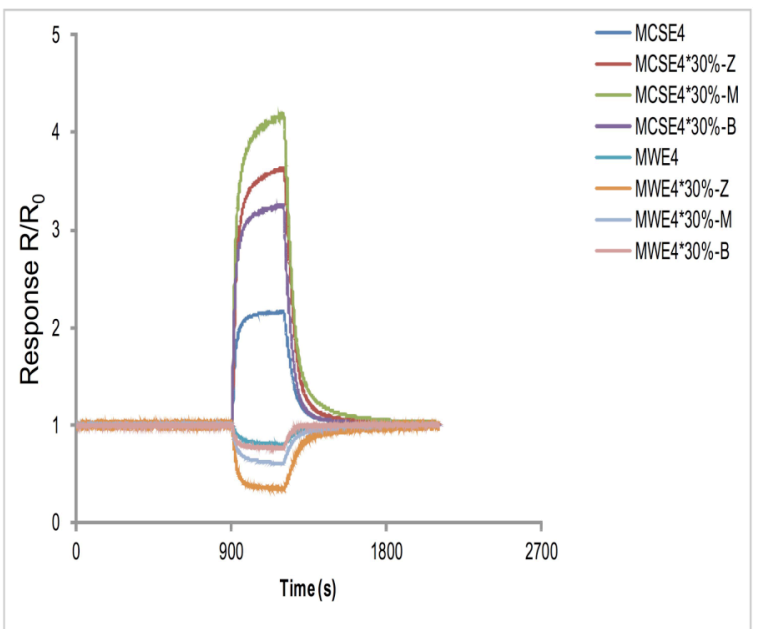

Fig. 4. Gas response behavior sensors with zeolite over-layers to $25 \mathrm{ppm}$ ethene in dry air at an operating temperature of $400{ }^{\circ} \mathrm{C}$. Where MCSE4 and MWE4 are chromium titanium oxide and tungsten trioxide sensors, admixed with $30 \%$ zeolite $Y-Z$, mordenite $-M$ or zeolite $B-B$.

All other zeolites gave a larger response than the control sensor (Fig. 3), indicating an acid catalyzed reaction to a product that $\mathrm{WO}_{3}$ is more sensitive too.

The gas response to $50 \mathrm{ppm}$ ethene of admixed zeolite sensors is presented in Fig 4. Similar phenomena can be observed that was seen with the zeolite over-layered sensors. In all cases the admixed sensors lead to a higher response than the control sensor alone and a generally faster response time. Significant discrimination across the array is observed. Responses of the sensors to $50 \mathrm{ppm}$ ethane, propene or butene are presented in Fig. 5 for the tungsten trioxide admixed sensors and Fig. 6 for the CTO admixed sensors.

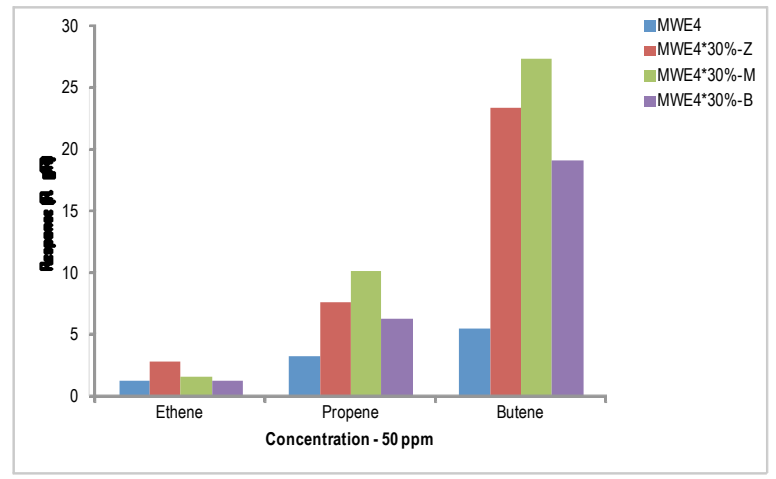

Fig. 5. Bar chart comparing response (conductive) magnitudes of the control and admixed $\mathrm{WO}_{3}$ sensors exposed to $50 \mathrm{ppm}$ of ethene, propene and butene in dry air at $400^{\circ} \mathrm{C}$.

In both cases higher responses are observed for the longer chain alkenes, this is unsurprising as a larger number of reactions can be expected to occur on the sensor materials surface.

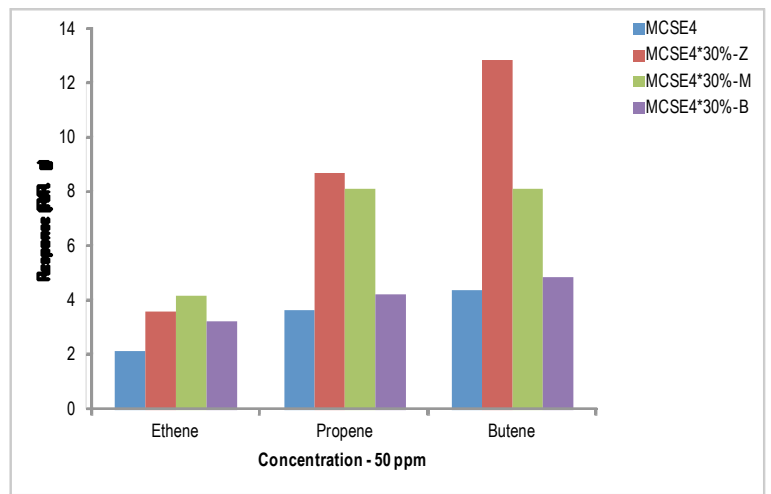

Fig. 6. Bar chart comparing response magnitudes of the control and admixed CTO sensors exposed to 50 ppm of ethene, propene and butene in dry air at 400 ${ }^{\circ} \mathrm{C}$.

The response patterns across the array are also significantly different in both magnitude and shape. Interestingly the response ratios change depending on the analyte gas, suggesting that there is a way to distinguish between otherwise similar gases. 


\section{Discussion}

Variables such as zeolite pore size, diffusion through the zeolite and to the sensor surface and the kinetics of surface reaction all play an important part in determining the overall sensor gas response. Certain reactions may occur within the entire sensor that can lead to an increase or decrease in the response of the system. Typically the response will be enhanced if such reactions lead to products that the sensor material has a higher sensitivity. The opposite being the case if the reactions form products that the sensor material is less sensitive to.

For the case of over-layered sensors the addition of a zeolite layer will primarily act to increase the response time (dependent on the diffusion of gas through the layer, Fig. 2), which will vary for different gas-zeolite combinations because of the size and shape of the molecular pores. A zeolite layer may also cause additional reactions to occur within the zeolite framework. Zeolites are well known for their catalytic properties and so reactions that increase or reduce the sensor response are both possible as are cracking reactions (effectively increasing the number of species present).

In the case of the admixed sensors diffusion affects as the result of the zeolite are expected to be much less important (Fig. 4), meaning that catalytic reactions will make a larger contribution to the overall sensor response, which is indeed what we have observed (Fig. 5 \&6).

\section{Conclusions}

The sensors give good responses to a wide variety of gases. Over-layered and admixed zeolite modified sensors have been successfully shown to be more selective and discriminating than the plain control sensors. This is a result of a combination of the zeolite materials intrinsic size / shape, catalytic and diffusion properties. As such the use of zeolites to modify metal oxide semiconductor gas sensors represents significant progress in improving the selectivity and sensitivity of such devices and arrays.

\section{Acknowledgements}

RB is grateful to the Royal Society for the provision of a Dorothy Hodgkin Research Fellowship and the EPSRC for funding (Grant number EP/H005803/1).

\section{References}

[1] G. Korotcenkov, "Metal oxides for solid-state gas sensors: What determines our choice?" Materials Science \& Engineering B 139,1-23 (2007); doi:10.1016/j.mseb.2007.01.044

[2] S. Akbar, P. Dutta, C.H. Lee, "High-Temperature Ceramic Gas Sensors: A Review" International Journal of Applied Ceramic Technology 3, 302311 (2006); ); doi: 10.1111/j.17447402.2006.02084.x

[3] S.C. Naisbitt, K.F.E. Pratt, D.E. Williams, I.P. Parkin, "A microstructural model of semiconducting gas sensor response: The effects of sintering temperature on the response of chromium titanate (CTO) to carbon monoxide." Sensors \& Actuators B: Chemical 114, 969-977 (2006); doi: 10.1016/j.snb.2005.07.058

[4] P. Wan, L. Kong, X. Wang, J. Li, "The design of a new integrated gas sensor array based on FEA." Sensors \& Actuators B: Chemical 66, 66-69 (2000); doi:10.1016/S0925-4005(00)00369-5

[5] A. Abbas, A. Bouabdellah, "Theoretical description of the interactions between a mixture of vapors and a set of chemical sensors by variable temperature." Journal of Mathematical Chemistry 42,1023-1030 (2007); doi: 10.1007/s10910-006-9157-y

[6] R. Binions, H. Davies, A. Afonja, S. Dungey, D.W. Lewis, D.E. Williams, I.P. Parkin, "ZeoliteModified Discriminating Gas Sensors." Journal of the Electrochemical Society 156, J46 -J51, (2009); doi: 10.1149/1.3065436

[7] R. Binions, A. Afonja, S. Dungey, D.W. Lewis, I.P. Parkin, D.E. Williams "Discrimination effects in zeolite modified metal oxide semiconductor gas sensors" IEEE Sensors Journal 11, 11451151 (2011); doi: 10.1109/JSEN.2010.2084079

[8] P. Varsani, A. Afonja, D.E. Williams, I.P. Parkin, R. Binions "Zeolite-modified $\mathrm{WO}_{3}$ gas sensors Enhanced detection of $\mathrm{NO}_{2}$ " Sensors and Actuators B: Chemical 160, 475-482 (2011); doi: 10.1016/j.snb.2011.08.014

[9] G.F. Fine, L.M. Cavanagh, A. Afonja, R. Binions "Metal oxide semi-conductor gas sensors in environmental monitoring" Sensors 10, 54695502 (2010); doi: 10.3390/s100605469 